\title{
Coupled charge and spin dynamics in high-density ensembles of nitrogen-vacancy centers in diamond
}

\author{
R. Giri, ${ }^{1,}{ }^{*}$ F. Gorrini,,${ }^{1,2}$ C. Dorigoni, ${ }^{1}$ C. E. Avalos,${ }^{3}$ M. Cazzanelli,,${ }^{1}$ S. Tambalo,${ }^{1}$ and A. Bifone ${ }^{1}$ \\ ${ }^{1}$ Center for Neuroscience and Cognitive Systems, Istituto Italiano di Tecnologia, Corso Bettini 31, Rovereto, 38068, Italy \\ ${ }^{2}$ Dipartimento di Fisica, Università degli Studi di Trento, via Sommarive 14, 38123 Povo, Italy \\ ${ }^{3}$ Institut des sciences et ingénierie chimiques, Ecole Polytechnique Fédérale de Lausanne, 1015 Lausanne, Switzerland
}

(Received 13 January 2018; revised manuscript received 29 May 2018; published 2 July 2018)

\begin{abstract}
We studied the spin depolarization of ensembles of nitrogen-vacancy (NV) centers in nitrogen-rich singlecrystal diamonds. We found a strong dependence of the evolution of the polarized state in the dark on the concentration of NV centers. At low excitation power, we observed a simple exponential decay profile in the low-density regime and a paradoxical inverted exponential profile in the high-density regime. At higher excitation power, we observed complex behavior, with an initial sharp rise in the luminescence signal after the preparation pulse followed by a slower exponential decay. Magnetic field and excitation laser power-dependent measurements suggest that the rapid initial increase of the luminescence signal is related to recharging of the nitrogen-vacancy centers (from neutral to negatively charged) in the dark. The slow relaxing component corresponds to the longitudinal spin relaxation of the NV ensemble. The shape of the decay profile reflects the interplay between two mechanisms: the NV charge-state conversion in the dark and the longitudinal spin relaxation. These mechanisms, in turn, are influenced by ionization, recharging, and polarization dynamics during excitation. Interestingly, we found that charge dynamics are dominant in NV-dense samples even at very feeble excitation power. These observations may be important for the use of ensembles of NV centers in precession magnetometry and sensing applications.
\end{abstract}

DOI: 10.1103/PhysRevB.98.045401

\section{INTRODUCTION}

Nitrogen-vacancy (NV) centers in diamond have been explored in recent years for sensing applications (magnetic field, electric field, and temperature) [1-7], for quantum information processing [8,9], and as a source of hyperpolarization of ${ }^{13} \mathrm{C}$ and ${ }^{14} \mathrm{~N}$ nuclei [10-12] and nitrogen donor spins [13] in diamond and also in the nuclei outside the diamond [14]. The detection sensitivity of these systems is, in principle, limited by the spin-projection noise [15] and depends on many factors, including measurement contrast, detection efficiency, and the number of spin centers. If one considers only the number of spin centers, the detection sensitivity should scale as $\sqrt{n}$, where $n$ is the number of negatively charged $\mathrm{NV}$ centers $\left(\mathrm{NV}^{-}\right)$ [16]. Therefore, for sensing applications where sensitivity is a critical factor, ensembles of $\mathrm{NV}^{-}$centers are preferred over single $\mathrm{NV}^{-}$centers. In order to achieve high densities of NV centers, vacancies are generated in nitrogen-rich diamond using ion or electron irradiation followed by annealing at high temperatures $\left(>800^{\circ} \mathrm{C}\right)$. In addition to $\mathrm{NV}$ defects, irradiation and postirradiation annealing can create deep-level trap states such as neutral $\mathrm{NV}$ centers $\left(\mathrm{NV}^{0}\right)$ and charged nitrogen ions $\left(\mathrm{N}^{+}\right)$as well as divacancies [17]. These deep-level trap states influence the photophysics and spin relaxation of $\mathrm{NV}$ centers [18-20].

The spin-relaxation behavior of ensembles of NV centers in diamond has been studied as a function of NV density,

*rakshyakar.giri@iit.it magnetic field, and temperature [21,22]. At low NV density, the spin-relaxation rate was found to be strongly dependent on temperature, suggesting phonon-mediated relaxation as the dominant mechanism [21]. At high density, dipole-dipole interactions were shown to drive the relaxation of $\mathrm{NV}$ center polarization [23]. Moreover, additional spin-relaxation mechanisms have been proposed in dense ensembles of $\mathrm{NV}$ centers, including the formation of fast-relaxing centers which could depolarize the whole NV center ensemble [18,23]. It was hypothesized that the fast-relaxing centers are formed as a result of charge dynamics: ionization of $\mathrm{NV}$ centers $\left(\mathrm{NV}^{-} \rightarrow \mathrm{NV}^{0}\right)$ through a two-photon process (under intense excitation) $[24,25]$ and subsequent recharging $\left(\mathrm{NV}^{0} \rightarrow \mathrm{NV}^{-}\right)$ through electron hopping between defect sites [26]. Therefore, initialization with a weak laser pulse is preferred to avoid ionization-induced depolarization. Nevertheless, a weak laser pulse can still ionize the NV centers indirectly: ionization of neutral nitrogen $\left(\mathrm{N}^{0}\right)$ through a single-photon process and subsequent tunneling of electrons from photoexcited $\mathrm{NV}^{-}$to a nearby $\mathrm{N}^{+}$. The efficiency of this process largely depends on the presence of nitrogen [27]. However, charge-state conversion of dense ensembles of $\mathrm{NV}$ centers at a low light level and its influence on spin dynamics are not well understood. In all of the reported experiments, the spin relaxation in the dark consisted of one component that could be fit with a simple or stretched exponential function.

Here, we have investigated the longitudinal spin relaxation $T_{1}$ of ensembles of NV centers in a single-crystal yellow diamond $(\approx 200 \mathrm{ppm}$ of substitutional nitrogen and $\approx 10 \mathrm{ppb}$ of NV centers) and in a purple diamond with the same 
concentration of nitrogen but much higher (more than 1000fold) concentration of NV centers. At a low excitation power level, we observed an exponential decay of the luminescence signal in the yellow diamond, consistent with previous literature. Surprisingly, an inverted exponential profile was found in the purple diamond, with a sharp growth in the luminescence signal following the initialization pulse even at very feeble excitation power. We have investigated the origin of this behavior by measuring longitudinal spin relaxation at various magnetic fields and for different excitation laser powers. We propose a model including the effects of charge and spin dynamics to account for the unusual behavior observed at very high densities of NV centers. We find that the shape and characteristic times of the relaxation curve depend critically on the interplay between these two mechanisms.

\section{METHODS}

Two high-pressure, high-temperature (HPHT) singlecrystal diamonds cut along the (100) crystallographic direction are used in this work and were produced by Element Six. One sample, "yellow" (Y1), is a standard commercial type-Ib HPHT diamond. The other one, "purple" (X1), is a type-Ib HPHT diamond electron irradiated and annealed to increase the concentration of $\mathrm{NV}$ centers. We determined the substitutional nitrogen $\left(\mathrm{N}^{0}\right)$ concentration from the absorption coefficient of the feature at $1135 \mathrm{~cm}^{-1}$ in the IR absorption spectrum (not shown here), following the methodology of Woods et al. [28]. We estimated that both samples contain more than $200 \mathrm{ppm}$ of $\mathrm{N}^{0}$. We observed that the IR absorption spectrum of sample $\mathrm{X} 1$ presents a feature at $1450 \mathrm{~cm}^{-1}$ which corresponds to interstitial nitrogen $[29,30]$. This feature is usually found in type-Ib diamond irradiated with high fluence and annealed at high temperature. We estimated that sample $\mathrm{X} 1$ contains about $100 \mathrm{ppm}$ of interstitial nitrogen. In sample Y1, the presence of interstitial nitrogen was not detectable. From the absorption coefficient of the 637-nm line in the UV-visible absorption spectrum and using the calibration constants from Davies [31], we estimated that sample X1 contains $\approx 10 \mathrm{ppm}$ of $\mathrm{NV}^{-}$. Comparing the luminescence of the two samples, we determined that sample $\mathrm{Y} 1$ contains $\approx 10 \mathrm{ppb}$ of $\mathrm{NV}^{-}$.

We used a confocal microscope built in house to study the depolarization of $\mathrm{NV}$ center ensembles. A microscope objective with a numerical aperture (NA) of 0.65 was used to focus the excitation laser $(532 \mathrm{~nm}$, Coherent Verdi) to a focal spot of around $1 \mu \mathrm{m}$. The fluorescence from the NV centers was collected with the same objective, filtered by a series of lowand high-pass filters, and a single-photon-counting module (Excelitas, SPCM-AQRH-14-FC) detected the luminescence in the range of $620-750 \mathrm{~nm}$. The photon-counting module has a high linear dynamic range and low dark count. We attenuated the luminescence using ND filters down to the picowatt level to avoid nonlinearity in photon counting. We used a three-axis Helmholtz coil system to control the strength and direction of a static magnetic field. An acousto-optic modulator (AA Optoelectronics) produced the excitation laser pulses, and a programmable transistor-transistor logic (TTL) pulse generator (PulseBlaster ESR-PRO) was used to generate the pulse sequences. Some of the experiments reported in this work involve the use of microwave fields. Microwaves produced by
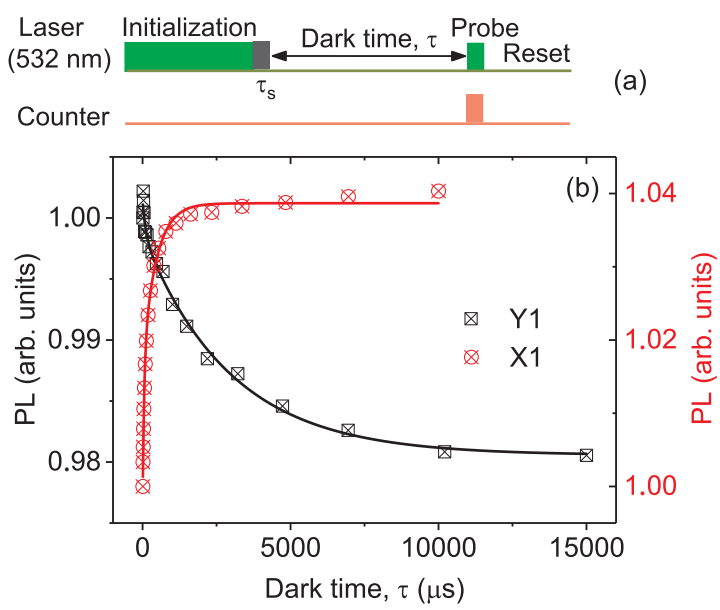

FIG. 1. (a) Pulse sequence used to study spin relaxation in the dark. Laser pulses of 500- $\mu$ s duration were used to initialize the NV centers, and probe pulses of $1-\mu$ s duration were used to read out the spin state after variable dark time. The dark time $\tau$ excludes the additional dark time $\tau_{s}=1 \mu \mathrm{s}$ introduced right after the initialization pulse to allow the spin populations in the singlet states to relax to the $m_{s}=0$ state. A reset time of $100 \mu \mathrm{s}$ was introduced to enable the system to reach equilibrium. (b) Spin depolarization in the dark: simple exponential decay profile in sample Y1 and an inverted exponential growth profile in sample X1. Solid lines are fits with Eq. (1).

an analog signal generator (Keysight Technologies N5171B) were passed through a high-isolation switch (Minicircuits ZASWA-2-50DR+) and were amplified using a 16-W amplifier (Minicircuits ZHL-16W-43-S+). A loop antenna of $1 \mathrm{~mm}$ diameter was used to deliver the microwave fields to the sample.

The NV defect ground state is a spin triplet with a zero-field splitting of $2.87 \mathrm{GHz}$ between the $m_{s}=0$ and $m_{s}= \pm 1$ sublevels. Optical pumping leads to an efficient spin polarization into the $m_{s}=0$ sublevel due to spin-dependent intersystem crossing towards an intermediate singlet state. The lifetime of the metastable singlet state varies from $460 \mathrm{~ns}$ at $4 \mathrm{~K}$ to $150 \mathrm{~ns}$ at $450 \mathrm{~K}$ [32-34]. The spin state can be read out through spin-dependent photoluminescence. After initialization into the $m_{s}=0$ state, the NV defect relaxes in the dark to a thermal equilibrium state, which is a mixture of $m_{s}=0, \pm 1$ states. The pulse sequence used for the measurement of the spin-relaxation time is shown in Fig. 1(a). The NV centers are excited by a green (532-nm) laser for $500 \mu$ s and probed for $1 \mu$ s after variable delay time $\tau$. The excitation and probing pulses are rather long compared to the ones used for single NV centers (a few-microsecond excitation and 300-ns readout pulses) $[35,36]$. The use of a longer pulse ensures efficient reinitialization of the NV ensemble [16,21,22], and the optimal pulse length largely depends on the experimental conditions such as the size of the NV ensemble, as well as excitation laser power (Fig. 8 in the Appendix).

An additional dark time $\tau_{s}=1 \mu \mathrm{s}$ was introduced right after the initialization pulse to allow populations in the metastable singlet state to decay towards the $m_{s}=0$ state. We introduced a reset time of $100 \mu \mathrm{s}$ after the probe pulse to allow the $\mathrm{NV}$ centers to reach charge equilibrium. In order to avoid differential charge-state buildup over many repetitions for 
different $\tau$ points, we ensured a constant duty cycle by cycling the acquisition over the entire curve. Moreover, we performed control experiments introducing a long reset time $(100 \mathrm{~ms})$, a lot longer than the typical relaxation times, to rule out any effect of variation in $\tau$. We also controlled for potential duty cycle effects by introducing a variable reset time to keep the sequence duration constant across $\tau$ points. The preparation pulse initializes the NV centers into the $m_{s}=0$ spin state, and relaxation of these NV centers in the dark was studied as a function of $\tau$. All of the experiments reported here were performed at room temperature, and the earth's field was not compensated for.

\section{RESULTS AND DISCUSSION}

Figure 1(b) shows the spin depolarization in both the samples used in this study, obtained with $200 \mu \mathrm{W}$ of excitation laser power at $B=0$, where $B$ is the external magnetic field. We observe that the depolarization curves are qualitatively different, with an exponential decay in the low-NV-density sample (decay time of the order of milliseconds) and a sharp increase in luminescence with a timescale on the order of $100 \mu \mathrm{s}$ in the high-NV-density sample. These decay profiles and timescales indicate that two distinct mechanisms may determine signal evolution in the two samples. The simple exponential decay profile and timescale on the order of milliseconds are similar to the reported longitudinal spin relaxation of NV ensembles of similar concentration [21,22]. However, the sharp increase in the luminescence signal is quite unusual and has not been reported in the literature.

The spin-depolarization curves measured as a function of excitation laser power are shown in Fig. 2. In the low-NVdensity regime (sample Y1), the decay profile deviates from the initially observed simple exponential-type relaxation, and a fast-rising component starts to develop and grows in amplitude with increasing laser power. In the high-NV-density regime (sample X1) we observed the reverse: the dynamics associated with the fast-rising component dominates at very feeble laser powers (down to $10 \mu \mathrm{W}$ ), and a slow-decaying component develops with increasing laser power. At high excitation power, the depolarization dynamics is qualitatively similar in both the high- and low-density regimes: a sharp increase in the luminescence signal following the preparation pulse followed by a slower exponential decay. This evidence further confirms the presence of two distinct mechanisms and suggests that both mechanisms may contribute to the signal evolution in the dark under different conditions. The fast-rising component is unlikely due to relaxation from the metastable singlet states, as was reported by Tetienne et al. [37]. The metastable-state decay time is $\approx 200 \mathrm{~ns}$ at $300 \mathrm{~K}$ [33], and in the experiments reported here, $1 \mu \mathrm{s}$ of additional dark time was introduced right after the initialization pulse to allow the spin populations in the metastable state to relax. Therefore, the sharp rise in the luminescence signal observed here (with a timescale of $\approx 100 \mu$ s) must be due to a different mechanism.

The green (532-nm) excitation laser pulse used in this study can ionize the $\mathrm{NV}$ centers $\left(\mathrm{NV}^{-} \rightarrow \mathrm{NV}^{0}\right)$ through a two-photon absorption process, and this process is more efficient at higher excitation power [24,25]. However, it has been suggested that ionization can occur even at low light
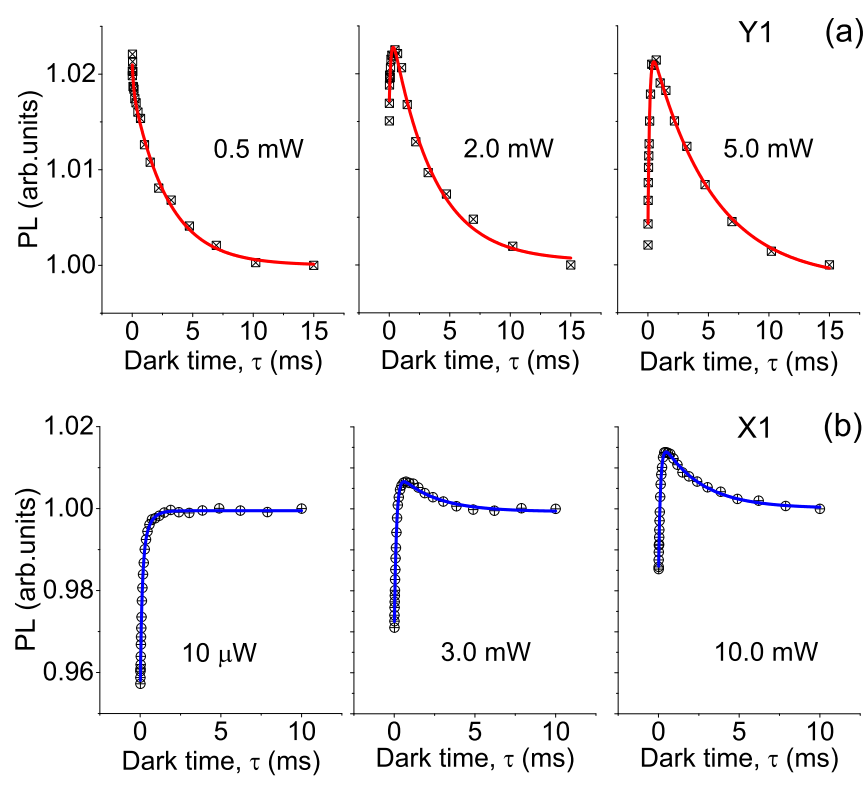

FIG. 2. Effect of excitation laser power. Spin relaxation in the dark at various excitation laser powers for (a) sample Y1 and (b) sample X1. In the yellow diamond (Y1), at low power, the decay profile is a single exponential, and a fast-rising component develops and grows in amplitude as a function laser power. In the purple diamond (X1), at low power, the luminescence signal increases sharply following initialization, and a slow-relaxing component builds up with increasing laser power. At high power, the decay profile appears to be qualitatively similar in both samples.

level via a tunneling process from a photoexcited $\mathrm{NV}^{-}$to a neighboring substitutional nitrogen $\mathrm{N}^{+}[13,27]$. These ionized $\mathrm{NV}$ centers can recharge $\left(\mathrm{NV}^{0}+\mathrm{N}^{0} \rightarrow \mathrm{NV}^{-}+\mathrm{N}^{+}\right)$and reach a charge equilibrium state. The recharging process can happen in the dark in the absence of any optical, electrical, or thermal excitation, and the mechanism suggested for this is the tunneling of electrons among closely spaced NV centers [23]. The recharging process could also be due to an impurity conduction process: electrons hopping between NV centers and nitrogen defects $\left(\mathrm{N}^{0}\right)$ [26]. This dynamics largely depends on the concentration and distribution of $\mathrm{N}^{0}$. If the concentration of $\mathrm{N}^{0}$ is high enough, then the availability of electrons to tunnel could facilitate recharging of NV centers in the dark [27]. A characteristic recharging time of around $100 \mu \mathrm{s}$ was found in a sample containing $45 \mathrm{ppm}$ of NV centers [23], which is close to the timescale observed in this study. The initial rise in the signal observed in our experiments is possibly related to this recharging process, and from now on we will refer to the corresponding time constant as the recharge time $T_{r}$.

All of the above observations suggest that the decay profile reported in this study could be a result of two competing processes pertaining to charge dynamics (ionization-recharge) and spin dynamics (polarization-relaxation).

We model the polarization-depolarization and ionizationrecombination dynamics by evaluating the populations within a simplified four-level scheme, as shown in Fig. 3. Ionization and recombination of the $\mathrm{NV}$ centers under various light levels also affects the spin populations in the $m_{s}=0$ state. We consider the ground-state triplet and a "metastable" level that includes 


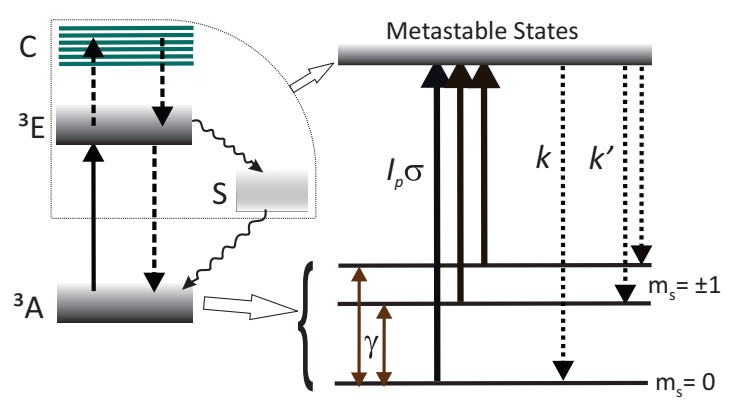

FIG. 3. Simplified energy levels of a NV center. ${ }^{3} A$ and ${ }^{3} E$ are the ground- and excited-state triplets, respectively. Label $C$ represents the conduction band, and label $\mathrm{S}$ includes all the singlet states. In the simplified four-level scheme, the metastable state includes the excited state, conduction band, and the singlet states.

the excited state, the conduction band, and the dark singlet states.

We define $T_{1}$ as the relaxation time of the populations in the $\left|m_{s}=0\right\rangle$ state and $T_{r}$ as the recharging time $\left(\mathrm{NV}^{0} \rightarrow \mathrm{NV}^{-}\right)$. Solving the rate equations for the populations, we can write the evolution of the photoluminescence (PL) signal as

$$
I(\tau)=I_{e q}\left[1-\alpha\left(I_{p}\right) e^{-\tau / T_{r}}+\beta\left(I_{p}\right) e^{-\tau / T_{1}}\right] .
$$

Here $I_{e q}$ is the PL at equilibrium, $1 / T_{r}=\left(k+2 k^{\prime}+I_{p} \sigma\right)$ is the recharging rate, and $1 / T_{1}=\left(3 \gamma+I_{p} \sigma\right)$ is the spinrelaxation rate; $\gamma$ is the decay rate from $|0\rangle$ to $| \pm 1\rangle, I_{p}$ is the laser intensity, $\sigma$ is the cross section for both absorption and ionization, and $k$ and $k^{\prime}$ are the charge capture rates. During initialization, the recharging rate increases linearly with laser intensity. The linear dependence of charge-state recovery on laser intensity during initialization results from the assumption that $\mathrm{NV}^{-}$centers lose electrons predominantly through a single-photon process. This assumption comes from the experimentally observed fact that charge dynamics is strong even at feeble excitation laser power $(10 \mu \mathrm{W})$ and is consistent with previous observations [20]. In the dark $\left(I_{p}=0\right)$, the recharging rate is solely dependent on the charge capturing rates, and the spin-relaxation rate depends on the decay rate $\gamma$. However, the amplitude of charge-state conversion $\alpha$ and the amplitude of spin dynamics $\beta$ depend on the excitation intensity, rate of charge capture, and rate of depolarization, i.e., $\alpha\left(\gamma, k, k^{\prime}, I_{p} \sigma\right)$ and $\beta\left(\gamma, k, k^{\prime}, I_{p} \sigma\right)$. So these two processes are not completely independent of each other, and the depolarization dynamics in the dark is determined by the relative weight of these two competing processes. We note that our model does not suggest anything about the physical mechanisms behind the recharging process in the dark.

In order to investigate our hypothesis that the shape of the decay profile is a result of two competing processes pertaining to charge-state dynamics and spin dynamics, we measured spin depolarization as a function of an external magnetic field (Fig. 4). Indeed, we expect the two mechanisms to depend differently on magnetic field strength and orientation. There are four groups of $\mathrm{NV}$ centers in diamond corresponding to four different symmetry-axis orientations. At zero magnetic field as well as magnetic field aligned along the (100) direction, transition frequencies of all the $\mathrm{NV}$ centers overlap, and
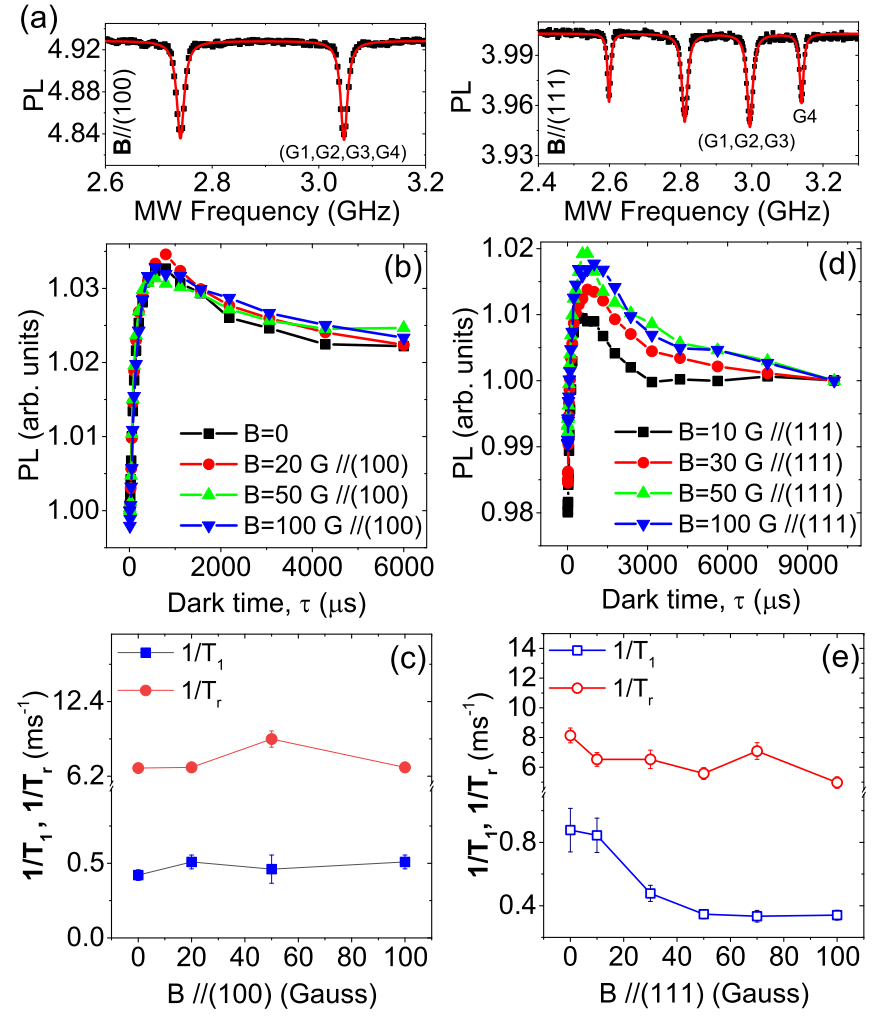

FIG. 4. Effect of external magnetic field. (a) Electron spin resonance spectra obtained from sample X1 for the case of magnetic field $B \|(100)$ when all four subgroups of NV centers are resonant (left) and for $B$ aligned along the (111) direction (right). In this case one group of NV centers (G4) is separated from the other three resonant groups (G1, G2, G3). PL decay profile in the dark for different values of (b) $B \|(100)$ and (d) $B \|(111)$. The recharging rate $\left(1 / T_{r}\right)$ and spin-relaxation rate $\left(1 / T_{1}\right)$ plotted as a function of (c) $B \|(100)$ and (e) $B \|(111)$. The recharging rate is not influenced by external field, whereas the spin-relaxation rate does depend on $B \|(111)$.

cross-relaxation is maximum. If the magnetic field is aligned along the (111) direction, then one group of NV centers (G4) has a transition frequency different from the other three groups (G1, G2, G3), which are still degenerate [Fig. 4(a)]. The detuning in transition frequencies can be increased by increasing the magnetic field strength, reducing the cross relaxation. We observe that the change in magnetic field strength strongly influences the depolarization rate of the slow-relaxing component. The depolarization rate is maximum at zero field and decreases slowly as a function of the magnetic field [Fig. 4(e)]. Therefore, the NV-NV cross-relaxation-induced magnetic noise is most likely responsible for the depolarization of the slow-relaxing component. Comparison of $\left(1 / T_{1}\right)$ in the low-density and high-density samples ( $\mathrm{Y} 1$ and $\mathrm{X} 1$, respectively) reveals a faster relaxation rate for the high-density sample [Fig. 5(a)]. Furthermore, when we do apply a magnetic field, we observe that the $\mathrm{X} 1$ and $\mathrm{Y} 1$ samples approach the same $T_{1}$ value as is typically observed for the case of ensembles at room temperature [21]. In this higher-field regime, the relaxation rate is dominated by intrinsic phonon interactions and should be independent of [NV], which is what we observe. Therefore, we can assign the slow-relaxing component to 


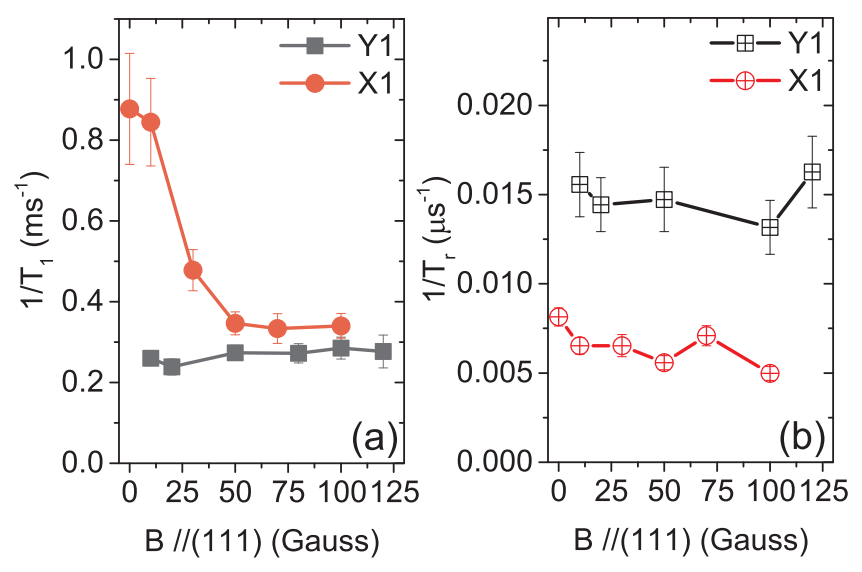

FIG. 5. (a) Spin-relaxation rate $\left(1 / T_{1}\right)$ and (b) recharging rate $\left(1 / T_{r}\right)$ as a function of $B \|(111)$. The spin-relaxation rate decreases with an increase in $B$ in the high-NV-density regime (X1), but the recharging rate is independent of $B$ in both the high- and low-NVdensity regimes.

the longitudinal spin relaxation $T_{1}$ of the NV centers. The sharp rising component is not affected by magnetic interaction, consistent with a charge-driven mechanism [Fig. 5(b)].

To corroborate the idea that the slow-decaying component is dominated by the longitudinal spin relaxation, we implemented another sequence dubbed the $T_{1}$ common-mode rejection technique designed to measure spin dynamics in high-NV-density samples [inset of Fig. 6(a)] [22]. This technique cancels out most of the background signals, as well as any contribution from charge-state conversion. The sequence begins with a $400-\mu$ s laser pulse, and after an adjustable dark time, another
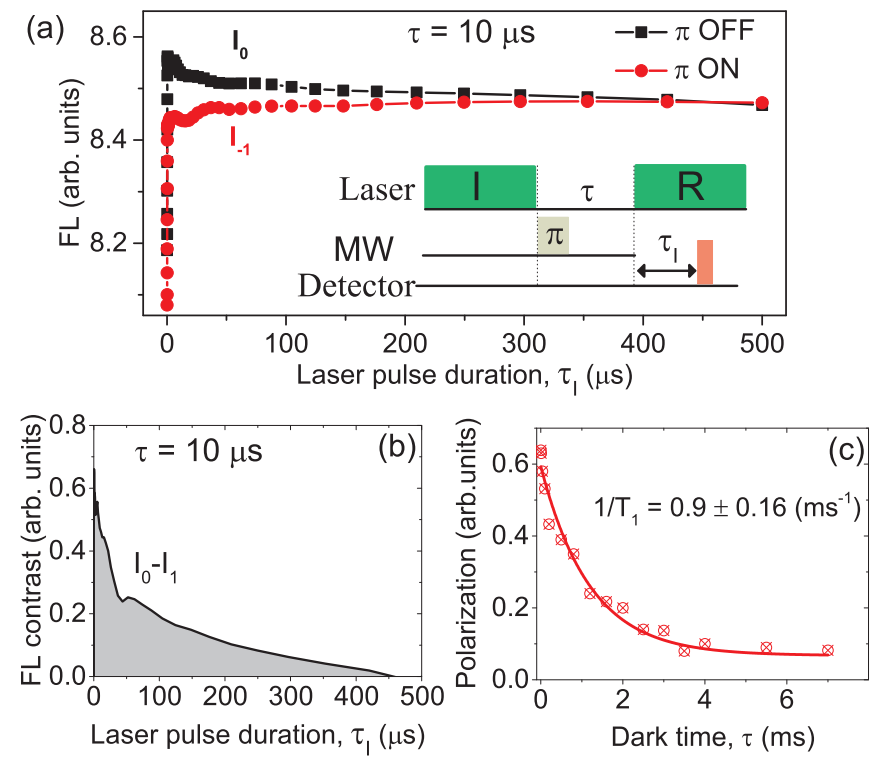

FIG. 6. $T_{1}$ common-mode rejection procedure. (a) Evolution of the NV populations in the $m_{s}=0$ and $m_{s}=-1$ states during the readout pulse, after waiting for $10 \mu \mathrm{s}$ in the dark following the preparation pulse. (b) The fluorescence difference between the two measurements with and without a $\pi$ pulse as a function of laser pulse duration. (c) Spin relaxation of the NV centers (excited by the microwave $\pi$ pulse) in the dark. laser pulse was applied to read out the remaining polarization of the NV centers. A microwave $\pi$ pulse right after the initialization pulse prepares the $\mathrm{NV}$ ensemble in the $m_{s}=-1$ state. The counter gate (width $=500 \mathrm{~ns}$ ) scans through the readout pulse to measure the remaining polarization of the $\mathrm{NV}$ centers in the $m_{s}=0$ and $m_{s}=-1$ states for each dark time. One such measurement is shown in Fig. 6(a) for dark time $\tau=10 \mu \mathrm{s}$. Fluorescence contrast $\left(I_{0}-I_{-1}\right)$ was evaluated for different dark times separately [Fig. 6(b)]. Residual spin polarization after each dark time was determined from the maximum amplitudes of the population difference and is presented in Fig. 6(c) as a function of dark time $\tau$. This technique measures only the residual spin polarization of the NV centers excited by the microwave $\pi$ pulse (i.e., $m_{s}=-1$ states in this case). One sees that the spin-relaxation rate obtained using this technique $\left(0.9 \pm 0.17 \mathrm{~ms}^{-1}\right)$ is comparable to the ones obtained from the biexponential decay curves as in Fig. 4. The spin-relaxation rates do depend on the initial spin state of the NV centers [23], and the slight difference in the spin-relaxation rates obtained using the two methods is due to the fact the alloptical method as described in Fig. 1 includes the background signals as well as the contribution from charge-state dynamics.

The results of magnetic-field-dependent measurements, as well as the $T_{1}$ common-mode rejection technique, corroborate the idea that that longitudinal spin relaxation indeed dominates the slow-relaxing component. However, we do not exclude other possible contributions to the slow-relaxing component.

The dynamics of ionization, recharging, and polarization during excitation and the resulting effect on depolarization dynamics in the dark in the two regimes of low and high densities of NV centers can be described as follows.

Low-NV-density regime (sample Y1). In this case, both $\alpha$ and $\beta$ increase linearly with laser power, but the change in $\alpha$ is more prominent [Fig. 8(a)]. Indeed, at the lowest power, ionization through the two-photon process is inefficient, and the decay profile in the dark is a single exponential reflecting the dominant spin dynamics process during initialization [Fig. 2(a)]. As the excitation laser power increases, $\mathrm{NV}^{-}$centers are ionized, creating more $\mathrm{NV}^{0}$ centers $[38,39]$. The laser pulse also ionizes the nitrogen donors $[19,40]$, and these electrons can be captured by $\mathrm{NV}^{0}$ centers to form $\mathrm{NV}^{-}$. The rate of ionization and recharging process increases as the laser power increases. Increasing $\beta$ indicates an increase in the degree of polarization with laser power. When the laser pulse is switched off, the recharging process continues in the dark until a charge equilibrium is attained. These dynamics are reflected in the biexponential decay profile.

High-NV-density regime (sample X1). In this case, $\alpha$ appears to be constant; however, $\beta$ increases almost linearly with power [Fig. 7(b)]. Charge dynamics dominate even at the lowest power and appears to saturate [Fig. 2(b)]. The strong charge dynamics at low laser power is quite unusual. Usually at low power, ionization is minimal because of the inefficiency of the two-photon process, and spin polarization as high as $90 \%$ can be achieved [24,41-43]. But here the rate of polarization is low in comparison to the ionization-recharging process. We must note that this sample is highly disordered with more than $200 \mathrm{ppm}$ substitutional $\mathrm{N}^{0}, 10 \mathrm{ppm}$ of $\mathrm{NV}^{-}$centers, and $100 \mathrm{ppm}$ of nitrogen occupying the interstitial sites. The interstitial nitrogen could be charged $\left(\mathrm{N}^{+}\right)$[29] or neutral 

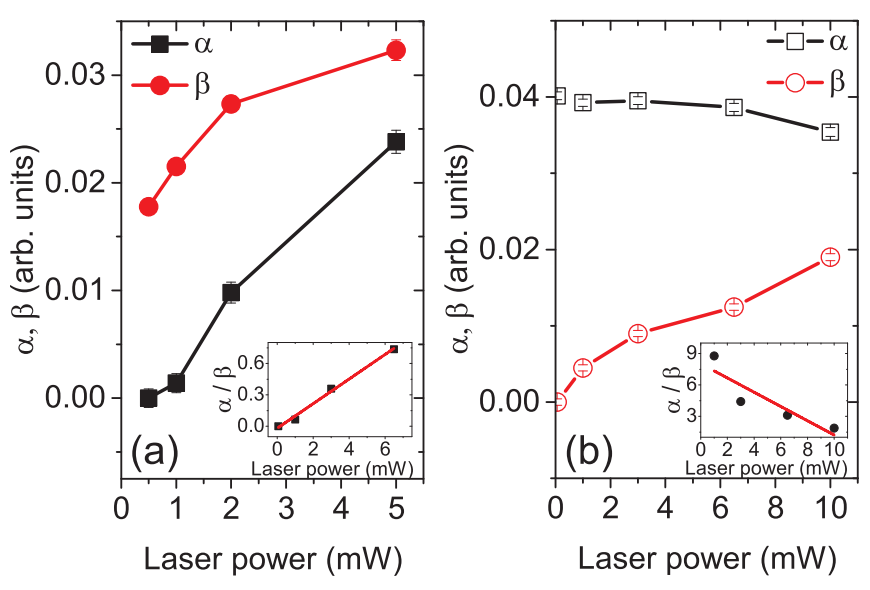

FIG. 7. Amplitudes of charge-state conversion $\alpha$ and spin dynamics (polarization-relaxation) $\beta$ as a function of excitation laser power in samples (a) Y1 and (b) X1. The solid lines are a guide to the eye. Insets: Linear dependence of $(\alpha / \beta)$ on excitation laser power.

$[30,43,44]$. It could also contain a significant number of other deep-level trap states such as divacancies [17]. It may also be possible that $\mathrm{NV}^{-}$centers exist in the form of $\left[\mathrm{NV}^{-}-\mathrm{N}^{+}\right]$pairs rather than as an isolated center [27]. Even at the lowest light level, the photoexcited $\mathrm{NV}^{-}$centers could lose electrons to the charged nitrogen $\left(\mathrm{N}^{+}\right)$through tunneling $\left(\left[\mathrm{NV}^{-}-\mathrm{N}^{+}\right] \rightarrow\right.$ $\left.\left[\mathrm{NV}^{0}-\mathrm{N}^{0}\right]\right)[13,27]$. Ionization of nitrogen donors $\left(\mathrm{N}^{0}\right)$ is also efficient, resulting in abundant electrons in the conduction band. These conduction electrons can be trapped by $\mathrm{NV}^{0}$ to form $\mathrm{NV}^{-}:\left(\left[\mathrm{NV}^{0}-\mathrm{N}^{0}\right] \rightarrow\left[\mathrm{NV}^{-}-\mathrm{N}^{+}\right]\right)$. Therefore, the ionization-recharging process is robust even at low laser power. As the excitation power increases more $\mathrm{N}^{0}$ are ionized, and trapping states are filled with electrons, resulting in depletion of the $\mathrm{N}^{0}$ reservoir [20]. We assume that the ionization of the electrons trapped in deep-level defects is slower than ionization of the donor electrons, so the charge dynamics slows down and reaches an equilibrium, resulting in an increase in $\mathrm{NV}^{-}$density. We are in a regime where the pumping rate is slower than the spin-relaxation rate. Thus, the increase in laser power results in an increase in the degree of polarization of the $\mathrm{NV}^{-}$centers. The saturation of charge dynamics during pumping is reflected in the constant amplitude of charge-state conversion in the dark $\alpha$, and an increase in the degree of polarization as a function of laser power results in a linear increase in the amplitude of spin dynamics (polarization-relaxation) $\beta$.

\section{CONCLUSIONS}

In summary, we studied the spin depolarization of ensembles of NV centers in single-crystal diamond. At low excitation power, we observed a simple exponential-type decay in the low-NV-density regime but a sharp rise in the luminescence signal right after the initialization pulse in the high-NV-density regime. At higher excitation power, we observed complex behavior, with a sharp rise in the luminescence signal followed by a slower exponential decay. Experiments with varying excitation laser power and magnetic field provide evidence that these decay profiles are due to a complex process involving charge and spin dynamics during pumping as well as in the dark. Our analysis indicates that charge dynamics, possibly due to tunneling among a network of closely spaced NV centers or among NV centers and nearby nitrogen atoms, may be dominant in the high-density regime even at very low laser excitation power. In this context, interstitial nitrogen present in NV-dense diamonds may play a significant role, even though a more detailed and systematic investigation of the involvement of these defects is needed. Our results corroborate previously reported findings that recharging of NV centers can occur in the dark in the absence of any laser or microwave irradiation [23]. Due to the effects of charge-state conversion on spin-relaxation measurements, controlling charge dynamics is vital in the application of ensembles of NV centers as sensors or for hyperpolarization of nuclear spins. A deeper understanding of the effect of charge-state conversion will have a significant impact on the use of $T_{1}$-based sensing schemes.

\section{ACKNOWLEDGMENTS}

We thank Dr. M. Orlandi and Prof. A. Miotello of IdEA laboratory, University of Trento, for helping us with the measurement of UV-visible absorption spectra of diamond samples.

\section{APPENDIX: SPIN REPOLARIZATION DURING READOUT}

An external magnetic field [50 G $\|(100)]$ was applied to split the \pm 1 level of the NV centers. We determined the frequencies $\nu_{ \pm 1}$, corresponding to the transitions $(0 \rightarrow \pm 1)$ from the optically detected magnetic resonance spectra. Microwave pulses ( $\tau$ is variable) with frequency resonant with the transition $(0 \rightarrow-1)$ were applied, which leads to an oscillation of the NV populations between the 0 and -1 spin states (Rabi oscillations). We found that the spin states can be flipped from the 0 state to the -1 state by applying a 290 -ns microwave pulse, known as a $\pi$ pulse.

After determining the microwave $\pi$ pulse duration, we used the pulse sequence shown in Fig. 8(a) to study the
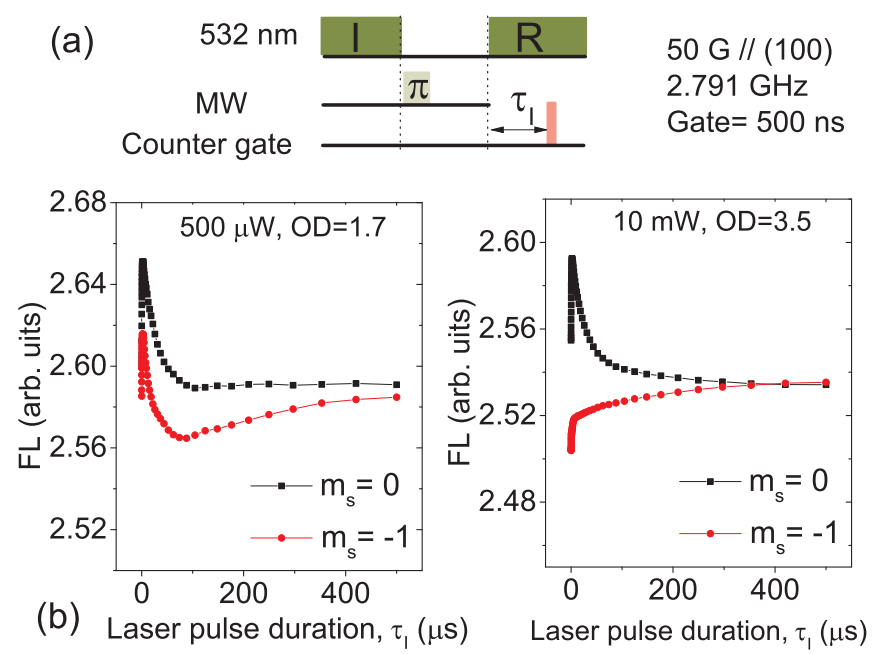

FIG. 8. NV spin repolarization during readout in sample X1. (a) Pulse sequence used to determine the optimal spin polarization and readout timescales. (b) Evolution of the fluorescence signal during the readout pulse for two different laser powers. 
repolarization dynamics. A 532-nm laser pulse and a microwave $\pi$ pulse prepare the NV centers in the 0 or -1 state. Spin repolarization of the NV ensemble was measured by scanning the photon-counter gate (500 ns) across the readout pulse. One sees that the maximum fluorescence contrast is obtained for a gate width/laser pulse duration of hundreds of microsec- onds, and the optimal gate width is laser power dependent [Fig. 8(b)]. These timescales are much larger than the typical timescales for a single NV center [35]. Thus, the optimal integration time for an NV ensemble depends on experimental conditions such as the size of the NV ensemble as well as excitation laser power and must be determined for each sample.
[1] R. Schirhagl, K. Chang, M. Loretz, and C. L. Degen, Annu. Rev. Phys. Chem. 65, 83 (2014).

[2] F. Dolde, H. Fedder, M. W. Doherty, T. Nobauer, F. Rempp, G. Balasubramanian, T. Wolf, F. Reinhard, L. C. L. Hollenberg, F. Jelezko, and J. Wrachtrup, Nat. Phys. 7, 459 (2011).

[3] L. Rondin, J. P. Tetienne, T. Hingant, J. F. Roch, P. Maletinsky, and V. Jacques, Rep. Prog. Phys. 77, 056503 (2014).

[4] M. Chipaux, A. Tallaire, J. Achard, S. Pezzagna, J. Meijer, V. Jacques, J.-F. Roch, and T. Debuisschert, Eur. Phys. J. D 69, 227 (2015).

[5] Y. K. Tzeng, P. C. Tsai, H. Y. Liu, O. Y. Chen, H. Hsu, F. G. Yee, M. S. Chang, and H. C. Chang, Nano Lett. 15, 3945 (2015).

[6] P. Neumann, I. Jakobi, F. Dolde, C. Burk, R. Reuter, G. Waldherr, J. Honert, T. Wolf, A. Brunner, J. H. Shim, D. Suter, H. Sumiya, J. Isoya, and J. Wrachtrup, Nano Lett. 13, 2738 (2013).

[7] J. P. Tetienne, A. Lombard, D. A. Simpson, C. Ritchie, J. Lu, P. Mulvaney, and L. C. Hollenberg, Nano Lett. 16, 326 (2016).

[8] L. Childress and R. Hanson, MRS Bull. 38, 134 (2013).

[9] R. Alleaume, F. Treussart, G. Messin, Y. Dumeige, J. F. Roch, A. Beveratos, R. Brouri-Tualle, J. P. Poizat, and P. Grangier, New J. Phys. 6, 92 (2004).

[10] R. Fischer, C. O. Bretschneider, P. London, D. Budker, D. Gershoni, and L. Frydman, Phys. Rev. Lett. 111, 057601 (2013).

[11] J. Scheuer, I. Schwartz, Q. Chen, D. Schulze-Sunninghausen, P. Carl, P. Hifer, A. Retzker, H. Sumiya, J. Isoya, B. Luy, M. B. Plenio, B. Naydenov, and F. Jelezko, New J. Phys. 18, 013040 (2016).

[12] R. Fischer, A. Jarmola, P. Kehayias, and D. Budker, Phys. Rev. B 87, 125207 (2013).

[13] M. Loretz, H. Takahashi, T. F. Segawa, J. M. Boss, and C. L. Degen, Phys. Rev. B 95, 064413 (2017).

[14] D. Abrams, M. E. Trusheim, D. R. Englund, M. D. Shattuck, and C. A. Meriles, Nano Lett. 14, 2471 (2014).

[15] V. M. Acosta, E. Bauch, M. P. Ledbetter, C. Santori, K.-M. C. Fu, P. E. Barclay, R. G. Beausoleil, H. Linget, J. F. Roch, F. Treussart, S. Chemerisov, W. Gawlik, and D. Budker, Phys. Rev. B 80, 115202 (2009).

[16] T. Wolf, P. Neumann, K. Nakamura, H. Sumiya, T. Ohshima, J. Isoya, and J. Wrachtrup, Phys. Rev. X 5, 041001 (2015).

[17] P. Deak, B. Aradi, M. Kaviani, T. Frauenheim, and A. Gali, Phys. Rev. B 89, 075203 (2014).

[18] X.-D. Chen, L.-M. Zhou, C.-L. Zou, C.-C. Li, Y. Dong, F.-W. Sun, and G.-C. Guo, Phys. Rev. B 92, 104301 (2015).

[19] H. Jayakumar, J. Henshaw, S. Dhomkar, D. Pagliero, A. Laraoui, N. B. Manson, R. Albu, M. W. Doherty, and C. A. Meriles, Nat. Commun. 7, 12660 (2016).

[20] J. Chen, S. Lourette, K. Rezai, T. Hoelzer, M. Lake, M. Nesladek, L. S. Bouchard, P. Hemmer, and D. Budker, Appl. Phys. Lett. 110, 011108 (2017).
[21] A. Jarmola, V. M. Acosta, K. Jensen, S. Chemerisov, and D. Budker, Phys. Rev. Lett. 108, 197601 (2012).

[22] M. Mrózek, D. Rudnicki, P. Kehayias, A. Jarmola, D. Budker, and W. Gawlik, EPJ Quantum Technol. 2, 22 (2015).

[23] J. Choi, S. Choi, G. Kucsko, P. C. Maurer, B. J. Shields, H. Sumiya, S. Onoda, J. Isoya, E. Demler, F. Jelezko, N. Y. Yao, and M. D. Lukin, Phys. Rev. Lett. 118, 093601 (2017).

[24] G. Waldherr, J. Beck, M. Steiner, P. Neumann, A. Gali, T. Frauenheim, F. Jelezko, and J. Wrachtrup, Phys. Rev. Lett. 106, 157601 (2011).

[25] N. Aslam, G. Waldherr, P. Neumann, F. Jelezko, and J. Wrachtrup, New J. Phys. 15, 013064 (2013).

[26] N. F. Mott, Can. J. Phys. 34, 1356 (1956).

[27] N. B. Manson and J. P. Harrison, Diamond Relat. Mater. 14, 1705 (2005).

[28] G. S. Woods, J. A. Van Wyk, and A. T. Collins, Philos. Mag. B 62, 589 (1990).

[29] G. S. Woods, Philos. Mag. B 50, 673 (1984).

[30] I. Kiflawi, A. Mainwood, H. Kanda, and D. Fisher, Phys. Rev. B 54, 16719 (1996).

[31] G. Davies, Physica B (Amsterdam, Neth.) 273-274, 15 (1999).

[32] N. B. Manson, J. P. Harrison, and M. J. Sellars, Phys. Rev. B 74, 104303 (2006).

[33] V. M. Acosta, A. Jarmola, E. Bauch, and D. Budker, Phys. Rev. B 82, 201202 (2010).

[34] L. Robledo, H. Bernien, T. van der Sar, and R. Hanson, New J. Phys. 13, 25013 (2011).

[35] M. Steiner, P. Neumann, J. Beck, F. Jelezko, and J. Wrachtrup, Phys. Rev. B 81, 035205 (2010).

[36] M. W. Doherty, N. B. Manson, P. Delaney, F. Jelezko, J. Wrachtrup, and L. C. Hollenberg, Phys. Rep. 528, 1 (2013).

[37] J.-P. Tetienne, T. Hingant, L. Rondin, A. Cavaillès, L. Mayer, G. Dantelle, T. Gacoin, J. Wrachtrup, J.-F. Roch, and V. Jacques, Phys. Rev. B 87, 235436 (2013).

[38] X.-D. Chen, C.-L. Zou, F.-W. Sun, and G.-C. Guo, Appl. Phys. Lett. 103, 013112 (2013).

[39] I. Meirzada, Y. Hovav, S. A. Wolf, and N. Bar-Gill, arXiv:1709.04776.

[40] F. J. Heremans, G. D. Fuchs, C. F. Wang, R. Hanson, and D. D. Awschalom, Appl. Phys. Lett. 94, 152102 (2009).

[41] J. Harrison, M. Sellars, and N. Manson, Diamond Relat. Mater. 15, 586 (2006).

[42] M. Howard, J. Twamley, C. Wittmann, T. Gaebel, F. Jelezko, and J. Wrachtrup, New J. Phys. 8, 33 (2006).

[43] S. Felton, B. L. Cann, A. M. Edmonds, S. Liggins, R. J. Cruddace, M. E. Newton, D. Fisher, and J. M. Baker, J. Phys.: Condens. Matter 21, 364212 (2009).

[44] A. Mainwood, Diamond Relat. Mater. 8, 1560 (1999). 